\title{
LOS FACTORES EXTERNOS EN LA TRANSFORMACIÓN DE LA FACULTAD DE DERECHO DE LA UNIVERSIDAD DE GUANAJUATO
}

\section{Sumario:}

1. Introducción. 2. La educación como un medio de respuesta social. 3. Los elementos o factores externos en la transformación de la otrora Facultad de Derecho de la Universidad de Guanajuato. 4. Algunos elementos externos que propiciaron la transformación. 5. Finalidad de la transformación.

Resumen: Este articulo trata de la transformación que ha tenido lugar en la División de Derecho, Política y Gobierno con motivo del impacto que los factores externos, como son la demanda de espacios de educación superior y la apertura de nuevos programas académicos, han originado que el sistema educativo se modernice para efecto de responder a las nuevas demandas sociales.

Palabras clave: factores, externos, educación, transformación, demanda, modernización, necesidad, social.

Abstract: This article describes the transformation being made at the former Law School in the University of Guanajuato. With the impact of external factors such as: the necessity of more educational spaces and the opening of new academic programs, the antique law school has changed its name; now is the Law, Politics and Government Division which involves three academic programs: law, public administration and political science designed to satisfy the new social needs.

Keywords: factors, external, education, transformation, requirement, modernization, needs, social.

\section{INTRODUCCIÓN}

Empiezo con una cita de Lucio Mendieta y Núñez, hecha en su artículo denominado "Apuntes para la Historia de la Facultad de Derecho", su frase reza de la siguiente manera:

* Profesor en la División de Derecho, Política y Gobierno del Campus Guanajuato de la Universidad de Guanajuato. 
"Una sintesis histórica de la Facultad de Derecho debe ser, para los abogados que de ella hemos salido, lazo de unión, motivo de solidaridad profesional; los grupos, las clases sociales, se unen no sólo por sus intereses, sino también por su pasado, del propio modo que los pueblos."2

Cuando recibí la invitación para participar con un artículo que de alguna manera dejara reflejada mi vivencia en nuestra majestuosa Universidad, y digo majestuosa, con el permiso de mis tres lectores, porque para mi eso es la Universidad de Guanajuato; una vez hecha esta acotación, me regreso; decía que cuando se me hizo la invitación, pasaron por mi mente innumerables recuerdos, de mis compañeros, de mis amigos, de mis maestros, de mis autoridades académicas, en fin de muchas personas, y me surgió la duda de lo que debería escribir y el formato que debería utilizar: artículo, reseña, reflexión o anécdota.

Dado que siempre he procurado conducirme con rigor académico, opté por tratar de escribir un artículo, teniendo como marco de referencia lo que representa transformar nuestra Universidad de Guanajuato, y específicamente lo que ahora es la División de Derecho, Política y Gobierno.

Sin embargo, el hecho de que adopte el formato de artículo, ello no necesariamente significa que dejaré de lado, la parte anecdótica y vivencial de mi paso como estudiante de licenciatura por la otrora Facultad de Derecho; y mucho menos, la transformación que ocurrió en mi persona con motivo de mi ingreso a la Facultad y de las repercusiones que tuvo en mi vida el haber gozado intensamente de sus aulas a la temprana edad de los 18 años. No haré referencia, para no aburrir a mis tres lectores, de mi experiencia en el estudio de la Especialidad en Notaria Pública; me circunscribiré a la licenciatura.

Además, me permitiré abordar este artículo teniendo como ejemplo el estilo del Maestro José Luis Requena, quien, escribió sobre su paso por la Escuela Nacional de Jurisprudencia de la UNAM, transformada posteriormente en la Facultad de Derecho. ${ }^{3}$

A efecto de acotar el presente trabajo de investigación, sólo se hará referencia al tema relacionado con los elementos externos que propiciaron la

\footnotetext{
2 Mendieta y Núñez, Lucio, “Apuntes para la Historia de la Facultad de Derecho", Manuales Jurídicos, Serie Número 1, UNAM, México, 2002, p. 7.

${ }^{3}$ Requena, José Luis, "Recuerdos de la Escuela Nacional de Jurisprudencia”, Serie Manuales Jurídicos, Número 3, Primera Edición, UNAM, México, 2002.
} 
transformación de la facultad a División. ¿Qué circunstancias rodearon su transformación? ¿Cómo se puede explicar la creación de la División desde la perspectiva de un alumno? Veamos.

\section{LA EDUCACIÓN COMO UN MEDIO DE RESPUESTA SOCIAL}

En toda la transformación que ha emprendido nuestra otrora Facultad de Derecho, saltan a la vista numerosas preguntas en torno a la necesidad social de transformarla para adecuarla a las nuevas necesidades, es decir, de la importancia que tiene el exterior, que pudiéramos denominarlo como el medio social, y la propia educación superior que se imparte en la Universidad de Guanajuato, que pudiera denominarse como: Universidad y Administración. Es decir, ¿cómo el medio social impacta a la Universidad y a su Administración que le exige transformarse? O en otros términos, ¿cuál es la relación que existe entre ambos órdenes uno interno y otro externo? "En ese sentido, las universidades deben definir claramente los límites de su régimen interno con el objeto de dar claridad y certidumbre a sus relaciones con el exterior...Solamente de esta manera se puede garantizar que la vinculación de las universidades con actores (y factores) externos se realice sin perjuicio ni menoscabo de los principios fundamentales que rigen su funcionamiento." 4

Indudablemente, las instituciones de educación superior, y específicamente la Universidad de Guanajuato debe impactar a la sociedad, debe responder a las necesidades y cambios que se den en la sociedad. El más visible, quizá, es el referido al incremento de la cobertura educativa, es decir, el abrir más espacios de educación superior, lo cual se puede lograr 1) a través del incremento de matrícula en los programas académicos existentes, o 2) a través de la creación de nuevos programas educativos.

Esta circunstancia externa no es nueva, ya ha acontecido en otros tiempos cuando la propia sociedad demandaba nuevos espacios o nuevas carreras, por ejemplo, la Segunda Guerra Mundial “... acercó a las universidades aún más hacia las necesidades nacionales...La entrada masiva de alumnos agudiza el cambio. Los nuevos roles exigen nuevos sistemas de organización y control, nuevos sistemas de gobierno." ${ }^{5} \mathrm{Y}$ así sucedió en tiempos anteriores, por ejemplo, con el abandono del modelo de las universidades medievales en donde el objeto de estudio se reducía a lo religioso; luego este modelo se transforma con Robert de Sorbon a la cabeza (creador de la Universidad de la Sorbona en Paris), en donde la universidad

\footnotetext{
${ }^{4}$ Sánchez Castañeda, Alfredo y Caballero Juárez, José Antonio, “La Vinculación en las Instituciones de Educación Superior y en las universidades: Autonomía y Sociedad", Instituto de Investigaciones Jurídicas de la UNAM, Serie Estudios Jurídicos, Num. 44, Primera Edición, México, 2003, p. 6.
} 
tenía como objetivo lograr una convivencia para la solución de problemas sociales; luego ocurrió lo que se conoció como el "movimiento Carnegie" que realizó un estudio económico de la educación (cuantos alumnos, cuantos programas académicos, recursos, etc.). Es decir, transformar a la otrora Facultad de Derecho es sólo un avance más en el desarrollo de la educación en el mundo.

En esta perspectiva, cuando el que esto escribe era estudiante de licenciatura (Generación 1990-1995), ingresaban a la otrora Facultad de Derecho un promedio de 45 estudiantes por semestre, solamente existía un programa académico: Licenciatura en Derecho, el único a nivel estatal en universidad pública. Esto deja en claro la enorme dificultad que se tenía de aprobar el examen de admisión y por lo tanto de ser alumno ordinario de dicha facultad. Al devenir del tiempo, sigo creyendo lo que en 1990 pensé y se quedó en mi mente: pertenecer a la Facultad es un privilegio. Recuerdo como si fuera ayer que presentamos el examen de admisión en el Auditorio General de la Universidad, completamente lleno, éramos más de 800 aspirantes, y al pensar que sólo pasarían 45 , se respiraban sentimientos encontrados de resignación y esperanza. Todos unidos en un mismo propósito pero con intereses contrapuestos.

Dada la necesidad de nuevos espacios educativos, y ya casi para terminar mi licenciatura, supe que se crearía otro programa académico dentro de la misma facultad: Licenciatura en Administración Pública, y que por lo tanto, el nombre de la Facultad también cambiaría a Facultad de Derecho y Administración Pública. Pues bien, con un nuevo programa, en mi opinión, se buscó satisfacer una demanda social de nuevos espacios educativos.

Así, al existir dos programas académicos, se incrementó la cobertura educativa de la Facultad, y con ello se abrió la posibilidad de estudiar algo distinto al Derecho para todos aquellos jóvenes que no habiendo pasado el examen de admisión a este programa académico, lo pudieran hacer en el otro. Esta no fue la finalidad, pero al menos, esa fue mi impresión. De cualquier manera, ya representaba una opción más para los jóvenes egresados de la preparatoria.

En estas vivencias, queda reflejado lo que señalé al principio: nuestra facultad estaba respondiendo a una necesidad social o elemento externo, entendido este como "...aquellos cuyas características están determinadas

\footnotetext{
${ }^{5}$ Ramírez Reynoso, Braulio, “Organización Académica y Administrativa de las Instituciones Públicas de Educación Superior", Serie Estudios Jurídicos Núm. 46, Instituto de Investigaciones Jurídicas de la UNAM, Primera Edición, 2003, p. 30.
} 
por el medio exterior, sin que las instituciones pueda modificarlos." ${ }^{6}$, o en otros términos, es el "...conjunto de elementos provenientes de la realidad social que actúan sobre los dos tipo de factores descritos anteriormente (internos e institucionales)."

\section{LOS ELEMENTOS O FACTORES EXTERNOS EN LA TRANSFORMACIÓN DE LA OTRORA FACULTAD DE DERECHO DE LA UNIVERSIDAD DE GUANAJUATO}

En este artículo sólo nos enfocaremos a los elementos externos que, se cree, orientaron la transformación de la otrora Facultad de Derecho (en tiempos de quien esto escribe) a transformarse en la División de Derecho, Política y Gobierno, todo envuelto en un halo de anécdotas del autor. Es de resaltar que ya en otras universidades del país como la Universidad Autónoma Metropolitana o la Universidad de Guadalajara existe una organización académica basada en Divisiones, en donde el Director de División es un funcionario "...que aparejan a su responsabilidad decisoria individualizada, la conducción ejecutiva de ámbitos colegiados formales dentro de los cuales son entes articuladores...".

La realidad que en 1990 imperaba era que la única universidad pública en el Estado que ofrecía el programa académico de Licenciatura en Derecho, lo era la Universidad de Guanajuato; en el Estado había, como las hay ahora, instituciones privadas de educación superior que ofrecían este programa académico, sólo por mencionar a la Universidad Iberoamericana, Campus León y a la otrora Universidad del Bajío (UBAC) que posteriormente se transformaría en la Universidad La Salle también en León, Universidad Quetzalcóatl en Irapuato, entre otras.

Esta realidad social o factor externo generaba que, como sucedió en mi caso, compitiéramos cientos de estudiantes de todos los rincones del Estado y algunos fuera del Estado, por los escasos 45 lugares disponibles, pues como ya quedó establecido, éramos más de 800 aspirantes, y entre otras cosas, el desconcierto reinaba entre nosotros al darnos cuenta de la enorme dificultad que existía por el número tan reducido de espacios para estudiar derecho, máxime si consideramos que ya el vivir en una ciudad distinta a la de origen, como fue mi caso, representaba un gasto para nuestros padres,

\footnotetext{
${ }^{6}$ Pallán Figueroa, Carlos, Bases para la Administración de la Educación Superior en América Latina: El Caso de México, ed. Instituto Nacional de Administración Pública, México, 1977, p. 29.

${ }^{7}$ Ibidem, p. 32.

${ }^{8}$ Ramírez Reynoso, Braulio, “Organización Académica y Administrativa de las Instituciones Públicas de Educación Superior", op. cit., p. 81.
} 
muchos de mis compañeros con dificultades económicas estudiábamos en la capital de nuestro Estado.

Pues bien, ¿cuáles fueron los factores externos que propiciaron la transformación de nuestra otrora Facultad de Derecho, a lo que ahora es la División de Derecho Política y Gobierno? Empecemos por definir que se entiende por factores externos, estos según Carlos Pallán son el “Conjunto de elementos provenientes del sistema social -por tanto no modificables por la propia institución -de naturaleza política, jurídica, económica, sociológica, cultural, las cuales se constituyen en el marco donde se dan los tres factores anteriores (internos, institucionales e interinstitucionales)" ${ }^{9}$

Con el objetivo de no dejar ninguna definición sin clarificar baste decir que los factores internos son "...elementos determinados por la propia institución e integrados por una serie de normas, técnicas y procedimientos (actividades-medio) que constituyen el llamado proceso administrativo, cuya finalidad es la conducción eficiente de la organización"; ; ${ }^{10}$ los factores institucionales son aquellos que se encuentran "...determinados por...una serie de actividades docentes, de investigación, de extensión, culturales (actividades-fin), a través de las cuales la institución cumple con los fines esenciales para los que fue creada"; 11 mientras que por elementos interinstitucionales se encuentra que son aquellos que "...obedeciendo a realidades, criterios, leyes y motivaciones provenientes del medio social o (de la organización, ayudan a)....alcanzar los objetivos de cada institución y a través de relaciones funcionales, de complementación o coordinación" ${ }^{12}$

Una vez aclarados los conceptos, se prosigue con la anécdota. Al haber superado el examen de admisión, recuerdo que uno de los comentarios comunes entre los compañeros de generación era en el sentido de considerarnos, como ya dije, privilegiados al poder ingresar a estudiar derecho. El medio social, traducido en un elevado número de solicitudes para poder ingresar a estudiar el programa académico de la Licenciatura en Derecho, demandaba la existencia de más espacios, sobre todo cuando, los propios compañeros señalaban que debido a esta demanda social, escuelas particulares de educación superior se estaban enfocando a la apertura del programa académico de licenciatura en derecho, nosotros, en tono quizá hasta cierto

\footnotetext{
${ }^{9}$ Pallán Figueroa, Carlos, Bases para la Administración de la Educación Superior en América Latina: El Caso de México, op. cit., p. 35.

${ }^{10}$ Ibidem, p. 34.

${ }^{11}$ Ibidem.

12 Ibidem, p. 35.
} 
punto socarrón y burlesco, decíamos que eran "escuelas patito"; pues para los compañeros de generación, la apertura de escuelas de nivel superior privadas que ofertaran derecho, representaba, un nicho de oportunidad por la gran demanda social. Pues bien, ¿Cuáles fueron esos elementos externos que propiciaron la transformación?

\section{ALGUNOS ELEMENTOS EXTERNOS QUE PROPICIARON LA TRANSFORMACIÓN}

Cada escuela o facultad de la Universidad de Guanajuato, tiene su propia historia y su propia visión. Al programa de la licenciatura en derecho, como ya dijimos, se han aceptado un número reducido de alumnos en comparación a su demanda, situación que también no es única ni exclusiva de nuestra otrora facultad, sino que más bien entra dentro de un movimiento acaecido en las universidades mexicanas. Esta aseveración de ninguna manera pretende ser una justificación, sino más bien sólo una referencia a su origen; tan es así que se ha venido utilizando en la doctrina lo que se conoce como la metáfora del hospital. ¿En que consiste esta metáfora? "Es una reflexión que toca, indudablemente, a las puertas de muchas universidades mexicanas: lo que interesa no es tanto mejorar aún más a los "pacientes" sanos, sino curar a los enfermos. Se critica el que las universidades se enorgullezcan por rechazar un elevado número de estudiantes (a los que se tacha de deficientes mediante un test de dos horas) y por masacrar -esta parte es textual- a los alumnos que no alcanzan los estándares asignados."13

Siguiendo el mismo orden de ideas de Carlos Pallán, creo que algunos factores externos que impactaron al programa de la licenciatura en derecho obedecía, repito en mi época de estudiante, entre otras cosas, a lo siguiente:

a) "Fuerte incremento de las aspiraciones populares en materia educativa", ${ }^{14}$ específicamente en el área del derecho. Derivada de "un símbolo de status", o de un "consumo de viejas élites" (ejemplo: quiero estudiar derecho pues mi papá es abogado) o con la idea de que el estudio del derecho proporciona una "cultura general" de ahí que coloquialmente al abogado o licenciado en derecho se le diga "todólogo". Todo ello, repito siguiendo el orden de ideas de Carlos Pallán.

\footnotetext{
${ }^{13}$ Ramírez Reynoso, Braulio, “Organización Académica y Administrativa de las Instituciones Públicas de Educación Superior", op. cit., p. 32.

${ }^{14}$ Pallán Figueroa, Carlos, Bases para la Administración de la Educación Superior en América Latina: El Caso de México, op. cit, p. 35.
} 
b) "Aguda escasez de recursos que impide a las universidades públicas responder eficazmente a las nuevas demandas". ${ }^{15}$ Específicamente, es de todos conocido el reducido espacio físico (infraestructura académica) con el que cuenta nuestra otrora Facultad de Derecho, sin contar del tremendo esfuerzo que representa la contratación de nuevos profesores para lograr el incremento de la matrícula de este programa. "La libertad para contratar profesores es el fundamento básico para el establecimiento de la pluralidad dentro de la universidad. A través de esta prerrogativa las instituciones de educación superior pueden integrar su planta académica recurriendo exclusivamente a criterios académicos para la valoración de los candidatos." 16

c) "Inercia inherente a la propia universidad". ${ }^{17}$ Igualmente, de todos es conocido el famoso refrán que reza: "todo cambio genera resistencia". Es algo inherente a la propia estructura administrativa, es decir, cuando se quiere introducir un nuevo patrón de conducta en una organización, sea pública o privada, es natural que se generen nuevas necesidades y por lo tanto, se requieran nuevas actitudes del personal que permitan resolver con atingencia dichas necesidades, pero, en algunos casos, no todo cambio es visto como lo que es: una oportunidad de mejorar lo que hacemos. Consecuentemente, el proceso de cambio es lento, de progresiva maduración y de mejora constante en su implementación; parte esta última que representa el verdadero reto de una organización que desea transformarse, pues no es suficiente con plasmar las ideas de forma clara, precisa y concisa, sino que hay que llevarlas a la realidad: una verdadera implementación de una política pública.

d) "Inercia de la sociedad misma" ${ }^{18}$ Quizá en este apartado solo baste mencionar la siempre existente idea en la sociedad de que sus hijos sean abogados o médicos. Idea que tiene un importante arraigo popular.

Estos fueron algunos de los elementos externos que son comunes a las instituciones públicas de educación superior. En este apartado solo se mencionaron aquellos que tienen relación con el presente artículo y con las cir-

${ }^{15}$ Ibidem, p. 36.

${ }^{16}$ Sánchez Castañeda, Alfredo y Caballero Juárez, José Antonio, "La Vinculación en las Instituciones de Educación Superior y en las universidades: Autonomía y Sociedad", op. cit., p. 6.

${ }_{17}$ Pallán Figueroa, Carlos, Bases para la Administración de la Educación Superior en América Latina: El Caso de México, op. cit, p. 36.

${ }^{18}$ Ibidem. 
cunstancias propias a nuestro entorno. Con esta transformación, ¿Qué se persigue?; ¿Qué es lo que se busca? Ello lo estudiaremos en el siguiente apartado.

\section{FINALIDAD DE LA TRANSFORMACIÓN}

No tendría caso el emprender una idea sobre la transformación de lo que era la Facultad de Derecho, a lo que es la División de Derecho, Política y Gobierno, sin resaltar, puntualizar, reforzar el objetivo o finalidad de una comunidad específica como lo es la que se aglutina en torno a nuestra División.

Para el caso de nuestra Facultad, permítame el amable lector seguirle denominando así como vestigio a la época en la que estudié, el objetivo es muy claro y así se encuentra establecido en la exposición de motivos de la vigente ley orgánica: "incremento en cobertura". Así lo cita textualmente la exposición de motivos:

"La cobertura educativa universitaria es uno de los temas de mayor relevancia para la sociedad guanajuatense y por ende de quienes los representamos en este Poder Legislativo. Una de las preocupaciones más intensas y comunes en las familias de nuestra entidad, tiene que ver con el elevado número de jóvenes que no alcanzan lugar en el sistema pública de educación media superior y superior. ${ }^{\prime 19}$

Y sigue diciendo la exposición de motivos:

"La entidad enfrenta dos grandes retos: abatir el rezago educativo y crear los espacios educativos para una población en constante crecimiento. Ante estos hechos, es impostergable el imperativo de que la Universidad cuente con las condiciones que le permitan ampliar su oferta educativa con calidad y pertinencia, con el propósito de contribuir a que más guanajuatenses accedan a la educación superior, mejoren su calidad de vida y aporten sus capacidades al desarrollo del Estado". ${ }^{20}$

Es decir, la finalidad de la transformación de la otrora Facultad de Derecho en División de Derecho, Política y Gobierno, tiene una sustancia o fondo social, es decir, dar la oportunidad a que más estudiantes puedan

\footnotetext{
${ }^{19}$ Ley Orgánica de la Universidad de Guanajuato, Exposición de Motivos, “Normatividad Vigente de la Universidad de Guanajuato 2008”, ed. Universidad de Guanajuato, Primera Edición, Guanajuato, México, 2008, p.13.

${ }^{20}$ Ibidem.
} 
realizar sus estudios de educación superior, por ello, la transformación de nuestra facultad se equipara a la obtención de la autonomía universitaria, es decir, esta transformación, tal como sucedió con la obtención de la autonomía universitaria de nuestra alma Mater, “...no es, de ningún modo, un logro exclusivamente universitario, sino que es una conquista social del pueblo mexicano...". ${ }^{21}$

Pero además, la transformación de nuestra Facultad de Derecho, como ya se apuntaba, obedece a razones de oportunidad, es decir, con una idea profesionalizante hacia la sociedad, que permita que los nuevos problemas sociales sean resueltos con una perspectiva profesional, o siguiendo los términos expuestos por Leoncio Lara Saenz, quien es citado en la obra de Braulio Ramírez Reynoso “...una institución de educación superior debe proporcionar un tipo de formación que capacite al egresado para un enfrentamiento real y efectivo, dentro del desarrollo de sus actividades, con las necesidades que le presenta el medio social en que vive." 22

No olvidemos que "Los estudios superiores en México se operan de manera fundamental a través de dos tipos de organización académico-administrativa: por escuelas y facultades, y por departamentos académicos." 23 Es decir, la otrora Facultad de Derecho de la Universidad de Guanajuato abandona un sistema de organización académico-administrativa de tipo napoleónico para insertarse en un modelo matricial.

Cabe mencionar, sólo para efectos de puntualizar este apartado que "La estructura por facultades y escuelas, mayoritaria en el medio nacional de la educación superior, tiene como base a los programas, agrupando a los estudiantes en generaciones que siguen un mismo plan de estudios...La estructura departamental...agrupa a profesores e investigadores precisamente en un departamento que se desenvuelve en torno de un campo especializado del conocimiento... (a) la organización de grupos multidisciplinarios de investigación" ${ }^{24}$

El reto que tiene por delante nuestra facultad es enorme, pero con la colaboración y aportación de todos se cumplirá. El objetivo es claro: aumentar la cobertura educativa, ¿cómo se logrará? ¿Se aumentará el número

\footnotetext{
${ }^{21}$ Serrano Migallón, Fernando, “Jornadas de la Autonomía. Presente y Futuro de la Autonomía Universitaria”, Serie Manuales Jurídicos, número 25, Primera Edición, UNAM, México, 2005, p. 4.

${ }^{22}$ Ramírez Reynoso Braulio, “Organización Académica y Administrativa de las Instituciones Públicas de Educación Superior", Op. Cit., p. 150.

${ }^{23}$ Ibidem, p. 112.

${ }^{24}$ Ibidem, p.113.
} 
de programas educativos o se aumentarán el número de espacios disponibles en los programas existentes? Las respuestas en estas preguntas sólo el tiempo nos ayudará a responderlas, y sólo para terminar quisiera dejar patente mi agradecimiento y reconocimiento a todas aquellas personas que directa o indirectamente aportaron a mi educación, y de mis compañeros de generación, sólo puedo decir que sólo recuerdos buenos, chuscos, divertidos, y en algunos casos, apenantes, conservo de ellos. No terminaría de escribir todos esos buenos momentos, pues estas líneas solo representan una insignificante alusión al cambio radical que en mí se dio a partir del ingreso a mi querida Facultad de Derecho, la cual, ahora, me ha prodigado con su cuidado al honrarme con ser profesor desde hace, ya casi, 10 años. Pero esa, es otra historia. 
